\title{
A Near Infrared Spectroscopic Discrimination of Noodle Flours Using a Principal-Component Analysis Coupled with Chemical Information
}

\author{
Masanori KumagaI, ${ }^{*}, * \dagger$ Kikuko KarUbe,* Tomoaki SAto, ${ }^{*}$ Naganori OHISA,** \\ Toshio Amano, $* * *$ Ryoei KIKUCHI, $*$ and Nobuaki OGAWA* \\ * Faculty of Engineering and Resource Science, Akita University, Tegata Gakuencho, Akita 010-8502, Japan \\ **Akita Research Institute of Food and Brewing, Arayamachi, Akita 010-1623, Japan \\ ***OPT Research, Inc., Nihonbashi, Chuo-ku, Tokyo 103-0027, Japan
}

\begin{abstract}
Using a portable near infrared (NIR) spectrometer, we discriminated flours for making Japanese noodles (Soba), not only relying on a statistical and mathematical approach, but also on a chemical interpretation of the NIR spectra. In original NIR spectra, the particle-size difference, which results in an undesired systematic variation, was extracted and interpreted as the first-principal component factor by a principal-component analysis. The discrimination of flour materials cannot be satisfied by this factor. However, after a standardized treatment for the original spectra, the particle-size effects were eliminated; alternatively, differences in the chemical contents were extracted as principal-component factors. Using these factors, flour material discrimination was achieved much better. This study suggests a novel idea of utilizing the wavelength contribution ratio spectra for interpreting the factors extracted from the principal-component analysis for the NIR spectra. This report also describes the relationship between the NIR spectra and the chemical-analysis data.
\end{abstract}

(Received July 4, 2002; Accepted August 22, 2002)

\section{Introduction}

Near infrared spectroscopy (NIRS) has been developed and used as a rapid, nondestructive and convenient technique for the qualitative and quantitative compositional analysis of many kinds of materials. ${ }^{1,2}$ Development to the present originated with Norris and colleagues; they first applied NIRS to agricultural product analysis. ${ }^{3,4}$ Since then, NIRS has been recognized as a useful analytical method in a wide variety of fields which anticipate its growth potential for further development. We are also conducting a thorough investigation for NIRS feasibility, and have attained some positive results. ${ }^{5}$ In this work, we have attempted to distinguish flours for noodles using a portable NIRS instrument which relies not only on a statistical and mathematical approach, but also on a chemical interpretation of the NIR spectra. We suggest the novel idea of using the wavelength contribution ratio spectra to interpret factors extracted from the principal-component analysis for the NIR spectra. These wavelength-contribution ratio spectra facilitate the assignment of bands of group frequencies for the NIR spectra. We also describe the relationship between the NIR spectra and chemical-analysis data. Here, the flours were buckwheat flours and wheat flours. Soba, a popular Japanese noodle, is a thin brownish noodle made from buckwheat flour, or from a mixture of buckwheat flour and wheat flour. Wheat flour can be used as a binding agent and a texture modifier for Soba noodles. Therefore, there

\footnotetext{
† To whom correspondence should be addressed.

E-mail: kumagai@arif.pref.akita.jp
}

are many mixing ratios of these flours for Soba noodles. How can we demonstrate the possibility of distinguishing buckwheat flour and wheat flour using NIRS? The ultimate goal of this project is buckwheat flour content-ratio estimation in mixed flours.

\section{Experimental}

\section{Materials and methods}

Five kinds of buckwheat flours and ten kinds of wheat flours for making Soba noodles were obtained from a milling factory and investigated in this work. The NIR diffuse reflection spectra in the $1200-2400 \mathrm{~nm}$ wavelength region (800 data points) were measured three times for each sample with a portable near infrared spectrometer, PlaScan SH (OPT Research, Inc., Japan), ${ }^{5}$ equipped with an acousto-optical tunable filter (AOTF). It is widely believed that the AOTF method theoretically caused a wavelength shift. ${ }^{6}$ In the case of PlaScan SH, because polystyrene film is used to correct the wavelength at $1680 \mathrm{~nm}$, the shift from an accurate wavelength increases as it departs from the corrected wavelength. In this work, the wavelength was re-corrected using a grating monochromator device (NIRS 6500, FOSS-NIRSystems) for the apparent observed wavelength. A white ceramic disk was used as a reference in the diffuse reflection mode. Each flour sample was packed into a sample holder in which it was compressed against a quartz window. The moisture, protein, fat, and ash content of each sample were determined according to AOAC methods. ${ }^{7}$ The mean particle size of each sample was determined by a particle size analyzer (MICROTRAC HRA, 
Table 1 Chemical analysis data and particle size of 15 kinds of flour samples $(n=3)$

\begin{tabular}{|c|c|c|c|c|c|c|c|}
\hline \multicolumn{2}{|c|}{ Sample notation } & \multirow{2}{*}{$\begin{array}{c}\begin{array}{c}\text { Moisture } \\
(\mathrm{g} / 100 \mathrm{~g})\end{array} \\
14.26\end{array}$} & \multirow{2}{*}{$\begin{array}{c}\begin{array}{c}\text { Protein } \\
(\mathrm{g} / 100 \mathrm{~g})\end{array} \\
13.73\end{array}$} & \multirow{2}{*}{$\begin{array}{c}\begin{array}{c}\text { Ash } \\
(\mathrm{g} / 100 \mathrm{~g})\end{array} \\
1.86\end{array}$} & \multirow{2}{*}{$\begin{array}{c}\begin{array}{c}\text { Fat } \\
(\mathrm{g} / 100 \mathrm{~g})\end{array} \\
1.82\end{array}$} & \multirow{2}{*}{$\begin{array}{c}\text { Carbohydrate } \\
(\mathrm{g} / 100 \mathrm{~g})\end{array}$} & \multirow{2}{*}{$\begin{array}{c}\text { Particle size/ } \\
\mu \mathrm{m}\end{array}$} \\
\hline Buckwheat flour & B1 & & & & & & \\
\hline & B2 & 14.39 & 11.72 & 1.60 & 1.34 & 70.96 & 108.60 \\
\hline & B3 & 13.98 & 10.88 & 1.56 & 1.81 & 71.78 & 80.51 \\
\hline & B4 & 14.64 & 10.68 & 1.56 & 1.61 & 71.50 & 89.03 \\
\hline & B5 & 14.09 & 10.32 & 1.13 & 1.20 & 73.27 & 91.41 \\
\hline \multirow[t]{10}{*}{ Wheat flour } & W1 & 13.21 & 12.92 & 0.35 & 1.11 & 72.41 & 66.62 \\
\hline & W2 & 12.55 & 10.12 & 0.55 & 0.75 & 76.04 & 68.10 \\
\hline & W3 & 13.21 & 11.97 & 0.38 & 0.99 & 73.45 & 54.40 \\
\hline & W4 & 13.57 & 12.65 & 0.33 & 0.83 & 72.62 & 68.67 \\
\hline & W5 & 13.51 & 13.27 & 0.53 & 1.05 & 71.64 & 73.64 \\
\hline & W6 & 13.81 & 13.83 & 0.44 & 1.26 & 70.66 & 72.24 \\
\hline & W7 & 13.50 & 13.19 & .035 & 1.03 & 71.93 & 75.51 \\
\hline & W8 & 13.16 & 13.73 & 0.42 & 0.94 & 71.75 & 71.79 \\
\hline & W9 & 12.50 & 14.51 & 0.52 & 1.19 & 71.28 & 84.34 \\
\hline & W10 & 13.54 & 12.87 & 0.51 & 0.76 & 72.33 & 71.89 \\
\hline
\end{tabular}

Leeds \& Northrup, USA).

\section{Data analysis}

A software program (JUSE-Stat Works/V3.0, The Institute of Japanese Union of Scientists \& Engineers) was employed for a spectral data analysis. Because this program allows a maximum of 256 entries for data analysis, the 800 data points in the 1200 $2400 \mathrm{~nm}$ wavelength region were reduced to 200 data points by averaging four successive data points. All NIR data were subjected to a treatment by a Kubelka-Munk transformation ${ }^{8}$ and a standardized normalization procedure. ${ }^{9}$ The former treatment was applied to the original NIR spectra to correct for any non-linearity of diffuse reflectance data. Utilizing the Kubelka-Munk theory, the reflectance $(R)$ can be related to the absorption coefficient $(K)$ and scattering coefficient $(S)$; it is also related to the absorption $(A)$ by the following equation: ${ }^{10}$

$$
K / S=(1-R)^{2} / 2 R=\cosh A-1 .
$$

In general, the latter treatment, the standardized normalization procedure, is applied to remove any systematic variation; it reduces the effect of a baseline drift in the original NIR spectra. This treatment centers each spectrum around zero by subtracting the mean and dividing each value by the standard deviation of the entire spectrum. Spectra that have undergone a treatment by both procedures are referred to as the standardized spectra in this work. For a discrimination analysis, a principal component analysis (PCA) was applied to standardized spectral data.

\section{Results and Discussion}

\section{Characterization of materials}

Chemical-analysis data and the particle sizes of the 15 kinds of flour samples are listed in Table 1. The results of a t-test, which was performed to compare the means of the respective components between buckwheat flours and wheat flours, indicate a statistical significance for the moisture, ash, and fat content, and the particle size. For the protein and carbohydrate contents, there is evidence that the means are not different between buckwheat flours and wheat flours. In this work, all flour samples were for Soba noodles. Thus, the protein and carbohydrate contents are very important factors for noodle product characteristics; that is, these content ratios must be

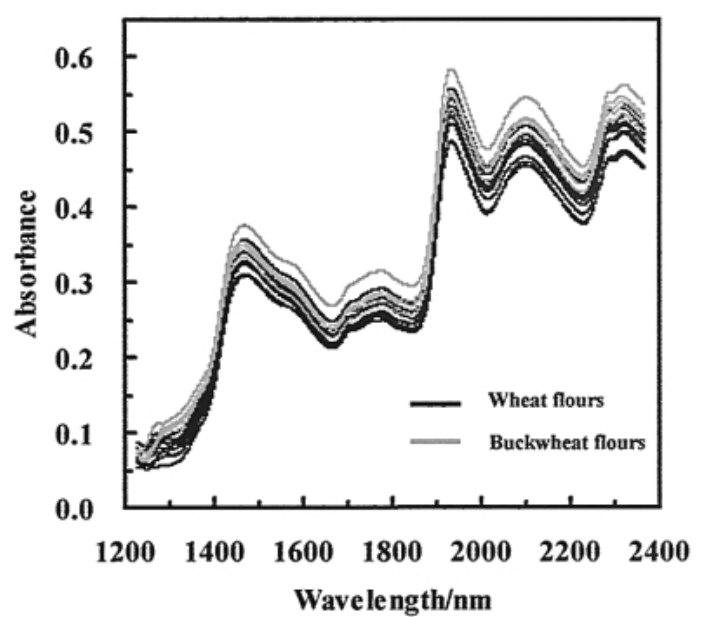

Fig. 1 Original near infrared spectra of 15 kinds of flour samples.

uniform within definite similar ranges for noodles.

Figure 1 shows the original NIR spectra for 15 kinds of flour samples. The spectra may contain some variance arising from scattering and spread, which gradually increase with the wavelength number. These observations account for the differences of the samples' powder surface in particle size, orientation, etc., which would induce variations in the diffuse reflection spectra, even if different portions of the same samples were measured. Consequently, to remove these extraneous systematic variations from nonscattering origins, the standardized normalization treatment was applied for the original NIR spectra. Before this standardized normalization treatment, a Kubelka-Munk transformation was performed, as mentioned above. For samples after the standardized treatment, the NIR spectra are illustrated in Fig. 2(a); plots of the individual absorbance versus the average absorbance are shown in Fig. 2(b). Only relatively narrow data spreads, compared to the original spectra, are essentially represented by the component differences of the investigated flour samples. In this manner, this procedure has a beneficial effect on multiplicative signal variances. However, it is difficult to distinguish buckwheat flours and wheat flours from Figs. 1 and 2. 

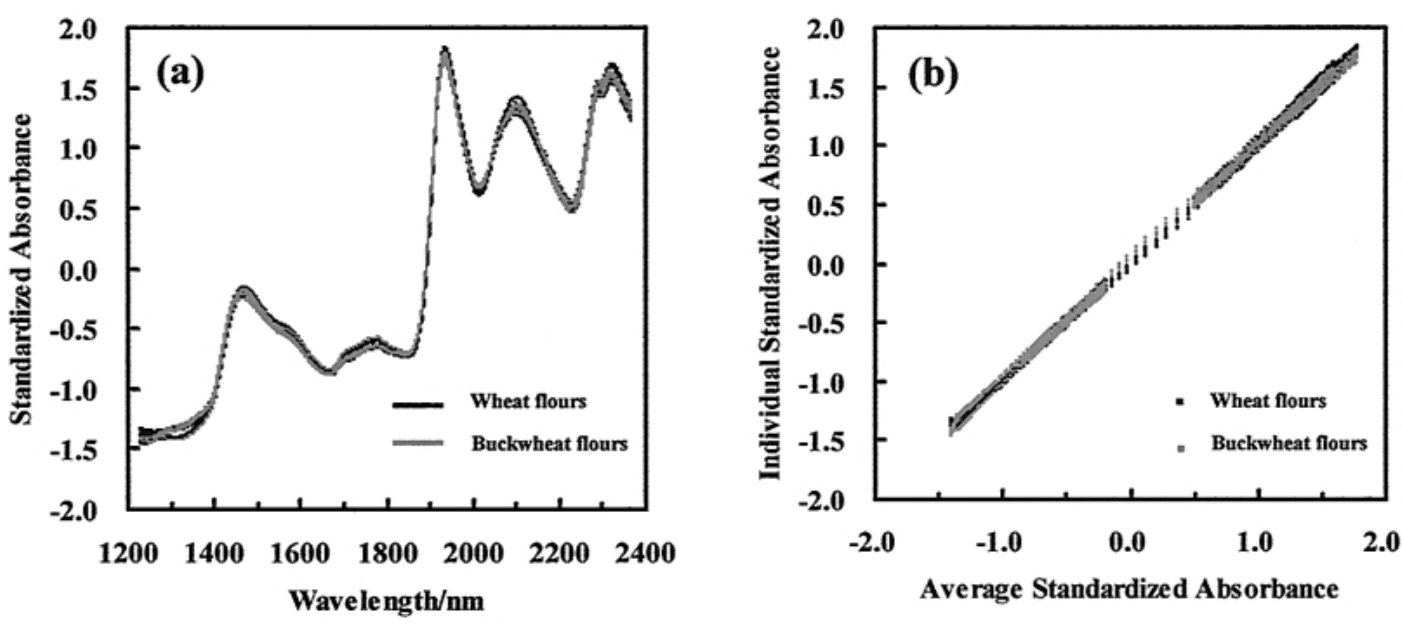

Fig. 2 Near infrared spectra after the standardized treatment (a) and plots of individual vs. average absorbance (b) of 15 kinds of flour samples.

(a)

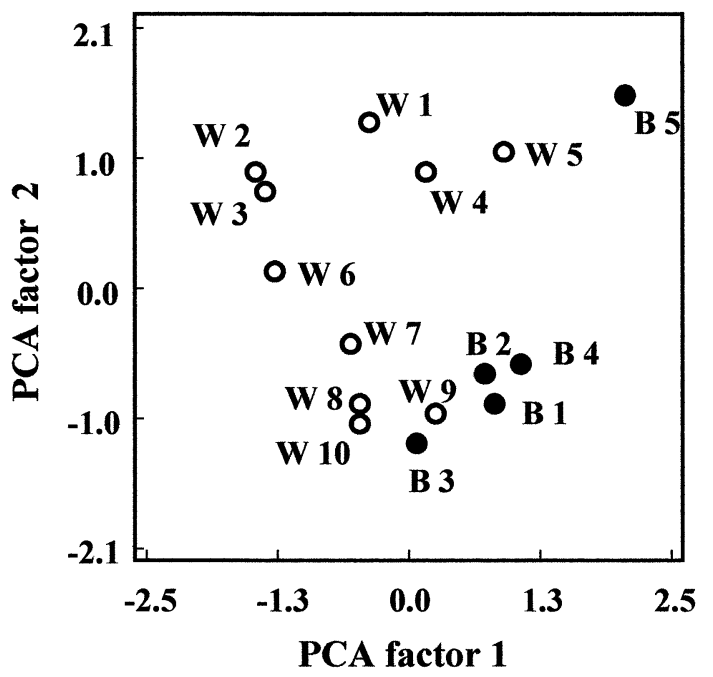

(b)

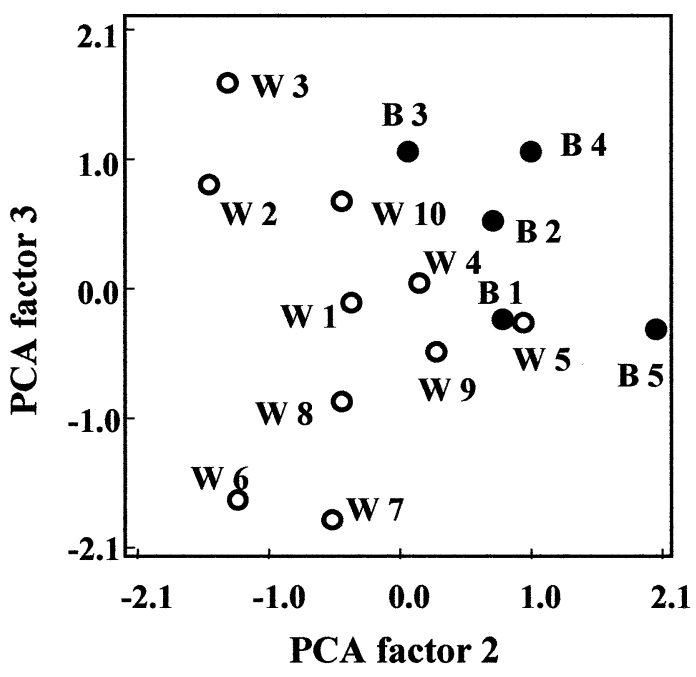

Buckwheat flour

Fig. 3 Score plots of PCA factor $1 v s$. factor 2 and factor $2 v s$. factor 3 for 15 kinds of flour samples based on original near infrared spectra.

\section{Principal-component analysis}

A principal-component analysis was applied to both the original and standardized NIR spectral data to reveal the sample characteristics more clearly. Figures 3(a) and 3(b) show score plots of the PCA factor 1 versus factor 2 , and factor 1 versus factor 3 for 15 kinds of flour samples based on the original NIR spectra. The first three principal components accounted for $87.8,6.2$ and $4.3 \%$ of the total variance, respectively. The separation between buckwheat flours and wheat flours was achieved with a few outliers, but it is not significant. However, Figs. 4(a) and 4(b), which are score plots based on standardized NIR spectra, show a clear distinction between them. For the case of the standardized spectra, the first three principal components accounted for $54.5,20.1$ and $10.7 \%$ of the total variance, respectively. The PCA factor 2 could be associated with discrimination.

To interpret PCA results, a correlation coefficient was calculated for the PCA factors and the chemical-analysis data, including the particle size of the samples. The results of a calculation for the original NIR spectra and after the standardized NIR spectra are listed in Table 2 as a correlation coefficient matrix. For the original spectra, the PCA factor 1 is generally correlated with the particle sizes. PCA factors 2 and 3 are generally correlated with the carbohydrate and protein content, respectively. On the other hand, after the standardized treatment, the effect of the particle size on PCA factor 1 vanished; consequently, PCA factor 1 generally correlated with the carbohydrate. PCA factor 2 roughly correlated with ash and protein; PCA factor 3 roughly correlated with the moisture. These results demonstrated that the effects of the standardized treatment mentioned above were very large in the original spectra. These results are in accordance with a multiplicative scatter correction (MSC), which was effective to reduce the influence of the particle size, as reported by Bertrand et al. ${ }^{11}$ 
(a)

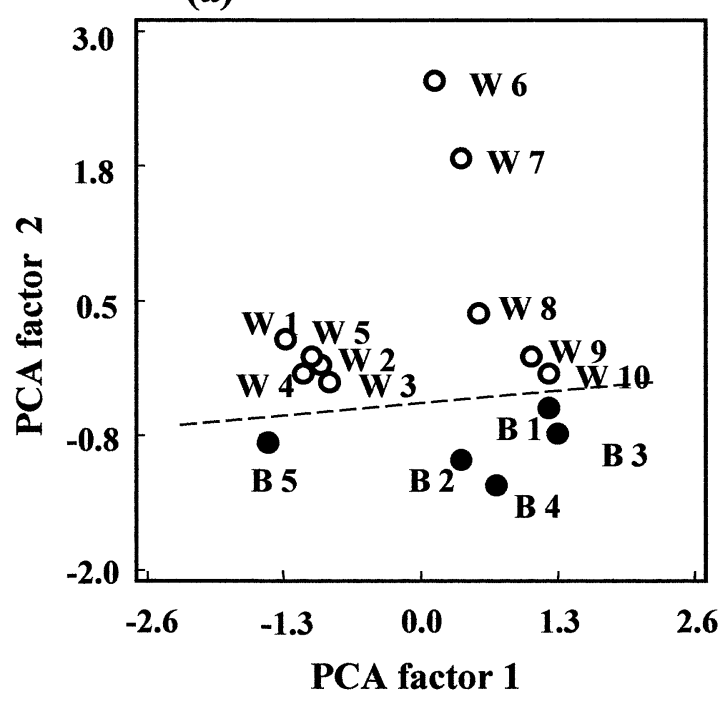

Wheat flour (b)

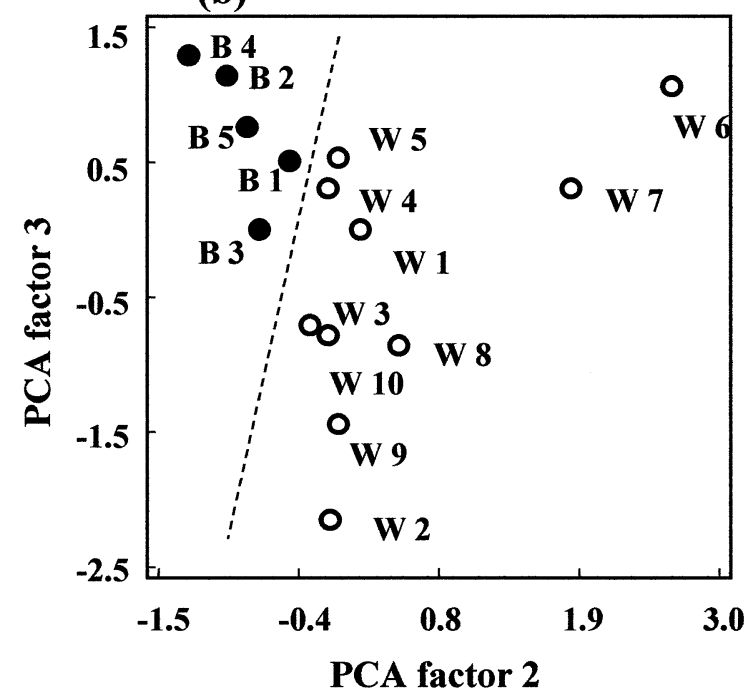

Buckwheat flour

Fig. 4 Score plots of PCA factor $1 v s$. factor 2 and factor 2 vs. factor 3 for 15 kinds of flour samples based on standardized near infrared spectra.

Table 2 Correlation coefficient matrix of chemical analysis data, particle size and PCA factors

\begin{tabular}{lrrrrrr}
\hline & \multicolumn{2}{c}{ Original spectra } & \multicolumn{3}{c}{$\begin{array}{c}\text { After the standardized } \\
\text { treatment }\end{array}$} \\
\cline { 2 - 7 } & PCA1 & PCA2 & PCA3 & PCA1 & PCA2 & PCA3 \\
\hline Moisture & 0.580 & -0.211 & 0.103 & 0.234 & -0.291 & 0.876 \\
Protein & -0.178 & -0.276 & -0.613 & 0.312 & 0.542 & -0.043 \\
Ash & 0.599 & -0.417 & 0.330 & 0.433 & -0.588 & 0.468 \\
Fat & 0.462 & -0.435 & 0.098 & 0.493 & -0.285 & 0.527 \\
Carohydrate & -0.367 & 0.546 & 0.348 & -0.602 & -0.090 & -0.562 \\
Particle size & 0.723 & -0.404 & -0.053 & 0.389 & -0.404 & 0.503 \\
\hline
\end{tabular}

Wavelength contribution ratio spectrum

We also showed that one could evaluate real meanings of the factor axes and associate the chemical information to the NIR spectra. Moreover, in order to examine the results of PCA, especially to interpret the factor axes, we calculated the wavelength contribution ratio. The contribution of the absorbance at each wavelength to the PCA factor was considered using the following formula:

Contribution ratio $(\%)=(\text { factor loadings })^{2} /$ (total number of wavelengths),

where factor loading indicates the correlation coefficient between the PCA factor and the original variable, which is for the absorbance at each wavelength.

Figure 5 shows the wavelength contribution ratio spectrum for PCA 1, which was generally correlated with the carbohydrate content, as shown in Table 2. The contribution ratio was highly correlated with starch, glucose and cellulose. A similar conclusion was derived for PCA 2 (Fig. 6) and PCA 3 (Fig. 7). That is, PCA 2 was generally correlated with the ash and protein, as shown in Table 2; because that contribution ratio is highly correlated with protein, the PCA 2 axis mainly denotes the protein content. On the other hand, PCA 3 is generally correlated with moisture, as shown in Table 2. While the contribution ratio is highly correlated with starch, the PCA 3

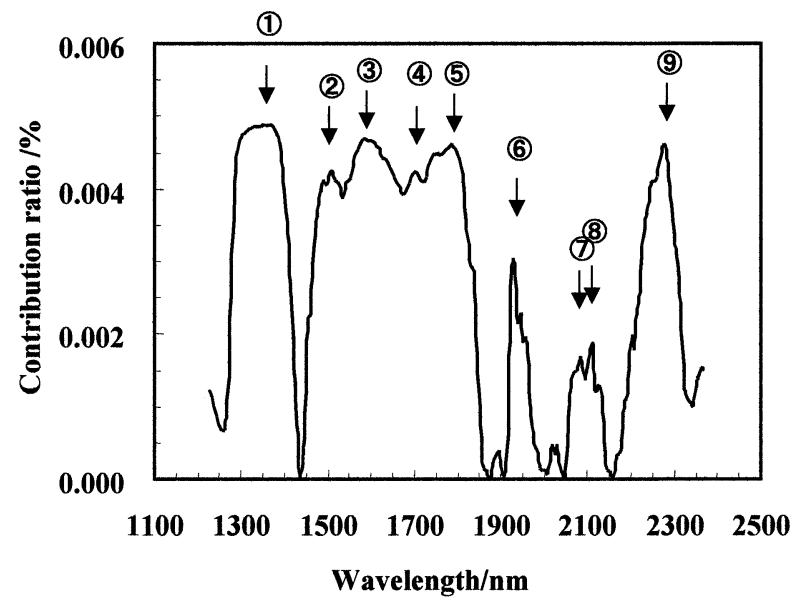

Fig. 5 Wavelength-contribution ratio spectrum for PCA 1. Peak assignment (band/nm): (1) $(1350-1400) 2 \times \mathrm{CH}$ str. $+\mathrm{CH}$ def. $\left(\mathrm{CH}_{3}\right.$, $\left.\mathrm{CH}_{2}\right)$; (2) (1480 - 1530) NH str. first overtone (-CONH-); (3) (1540) $\mathrm{OH}$ str. first overtone (cellulose); (4) $(1620-1765) \mathrm{CH}$ str. first overtone $\left(\mathrm{CH}_{3}\right)$; (5) (1780 - 1820) OH str. first overtone (cellulose, starch), OH str.+ $2 \times$ CO str. (cellulose, starch); (6) (1930) OH str. + $\mathrm{OH}$ def. (cellulose, starch); (7) (2080) OH str. + OH def. (sucrose, starch); (8) (2100) $2 \times \mathrm{OH}$ str. + $2 \times \mathrm{CO}$ str. (starch); (9) (2270 2280) $\mathrm{OH}$ str. + CC str. (cellulose).

axis could denote mainly the moisture content.

Relationship between the chemical-analysis data, particle size and NIR spectra

Figure 8 shows score plots of PCA factor 1 against factor 2 for 15 kinds of flour samples based on chemical-analysis data and particle size; it shows the orientation and amount of the factor loadings simultaneously. It is easy to signify dimensional space by overlapping the factor loadings as a vector expression on the score plots. Hence, buckwheat and wheat flours are isolated. This fact means that these flour samples can be discriminated by chemical-analysis data and particle size. From 


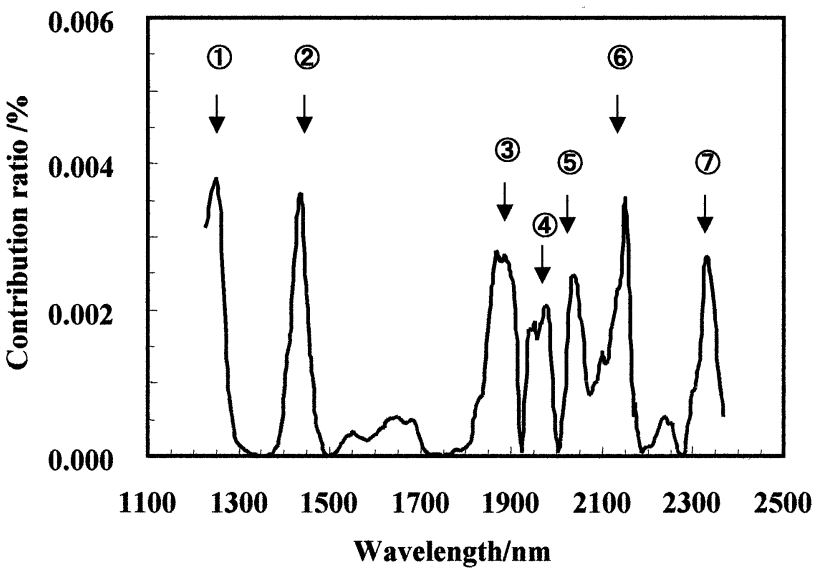

Fig. 6 Wavelength-contribution ratio spectrum for PCA 2. Peak assignment (band/nm): (1) (1250) $\mathrm{CH}$ str. second overtone $(\mathrm{CH})$; (2) (1440) $2 \times$ CH str. + CH def. (CH); (3) $(1860-1900) \mathrm{OH}$ str. $+2 \times$ CO str. (starch); (4) (1960 - 1990) NH sym str. + amide II (protein); (5) $\left(2030\right.$ - 2060) $\mathrm{CO}$ str. second overtone $\left(\mathrm{CONH}_{2}\right), \mathrm{NH}$ sym str. + amide II (protein); (6) (2130 - 2160) NH str. + CO str. (amino acid), 2 $\times$ amide I + amide III (CONH); (7) $(2330-2360)$ CH def. second overtone (cellulose).

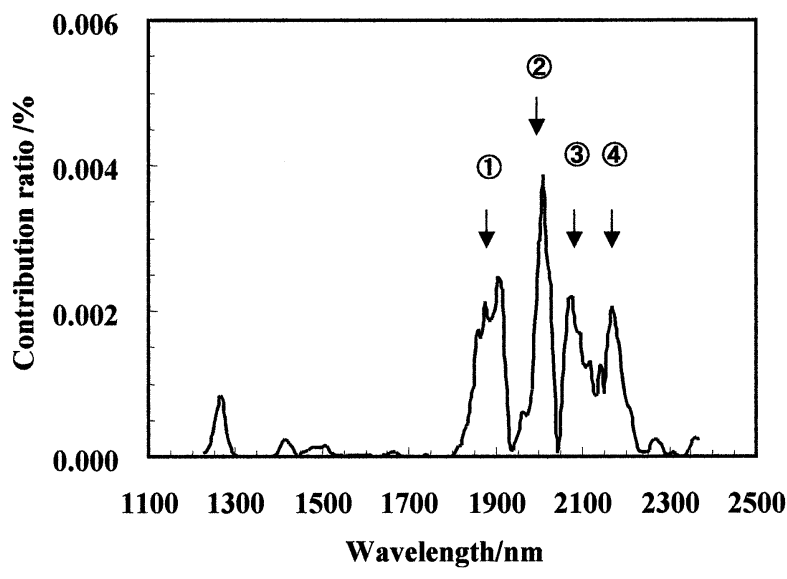

Fig. 7 Wavelength-contribution ratio spectrum for PCA 3. Peak assignment (band/nm): (1) $(1850-1920) \mathrm{OH}$ str. $+2 \times \mathrm{CO}$ str (starch), OH str. first overtone (ROH); (2) $(1990-2030) 2 \times \mathrm{OH}$ def. $+2 \times \mathrm{CO}$ str. (starch); (3) $(2060-2100) \mathrm{OH}$ str. $+\mathrm{OH}$ def. $(\mathrm{ROH}$, starch); (4) (2150 - 2190) $2 \times$ amide I + amide III $(\mathrm{CONH})$.

factor-loading information, the axes for discriminating these flours are classified into the three dimensions of proteins, carbohydrates, and the groups of fat, particle size, moisture, and ash contents. Although the carbohydrate and protein content differences between buckwheat flours and wheat flours are not statistically significant, these contents play the main role in distinguishing flours. Compositional differences among flour samples allow one to distinguish between buckwheat flours and wheat flours.

Furthermore, although it is regarded that the NIR spectra are overlapped by numerous broad bands of group frequencies, the NIR spectra simultaneously contain multiple compositional characteristics of samples. This fact indicates the superb functionality of NIRS; it also means that measuring of the NIR spectra makes it possible to distinguish flour types rapidly based on compositional differences without any time-consuming or complicated chemical-analysis. Especially, it is able to yield

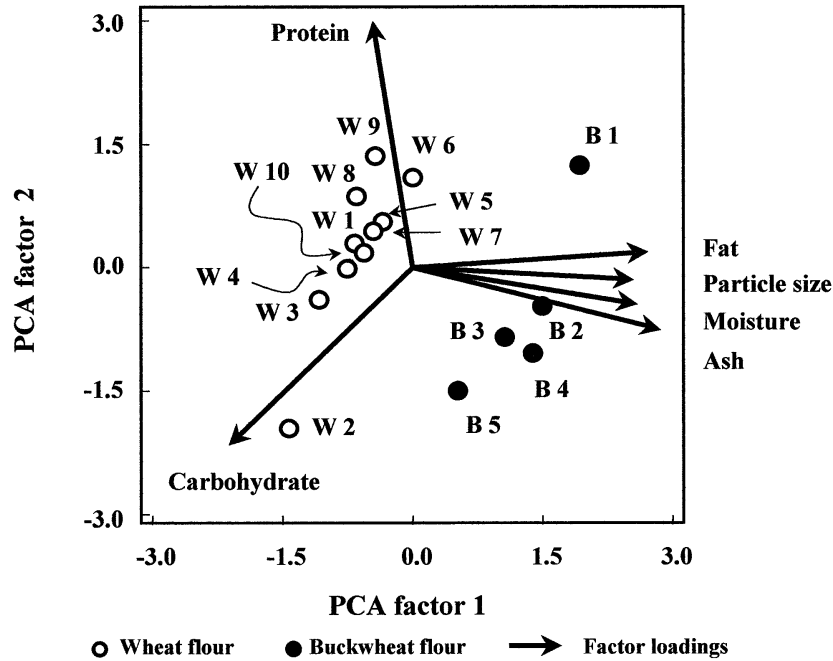

Fig. 8 Score plots of PCA factor $1 v s$. factor 2 for 15 kinds of flour samples based on chemical-analysis data and particle size.

discrimination results rapidly in the field using a portable NIR spectrometer, like the PlaScan discussed here.

\section{Conclusions}

Using a portable near infrared (NIR) spectrometer, we discriminated flours for making Japanese noodles (Soba) by relying not only on a statistical and mathematical approach, but also on a chemical interpretation of the NIR spectra. We assume the importance of a chemical interpretation of the spectra, which corroborates evidence based on the analytes' constituent materials. Accordingly, in the original NIR spectra, the particle size difference, which result in an undesired systematic variation, was extracted and interpreted as the first principal-component factor by principal-component analysis. Discrimination of flour materials cannot be satisfied by this factor. Influence of these systematic variations, which are not correlated with the property of sample composition, should be removed by a spectral correction. This work demonstrated that, after the standardized treatment for the original spectra, the particle-size effects were eliminated; alternatively, any differences of the chemical contents were extracted as principalcomponent factors. Using these factors, the discrimination of flour materials was achieved much better. Also, we emphatically suggest the novel idea of utilizing the wavelength contribution ratio spectra for interpreting factors extracted from the principal-component analysis for the NIR spectra. The wavelength contribution ratio spectra facilitate assignment of bands of group frequencies for NIR spectra. Although NIR spectra generally have broad bands overlapping some group frequencies, they may simultaneously contain multiple compositional characteristics of samples. In this work, we also showed that the NIR spectra were reflected in the sample compositional characteristics. This fact indicates the superb functionality of NIRS; it also means that measuring the NIR spectra makes it possible to distinguish various types of flour rapidly based on compositional differences without any timeconsuming methods, such as a complicated chemical-analysis. Notably, discrimination can be performed rapidly even outside of a laboratory using a portable NIR spectrometer, like the PlaScan used here. 
Our final goal was to estimate the buckwheat contents of noodles made from mixed flours prepared from buckwheat flours and wheat flours in varied mixing ratios. We first presented results showing that the discrimination of buckwheat flours and wheat flours was possible using respective NIR spectra. Our strategies are to propose a qualitative discrimination of materials coupled with chemical information based on their compositional differences; the method relies not only upon a chemometrical approach, but also on chemical interpretation of the NIR spectra. In the future, we would like to discern the buckwheat content in the Soba noodles, themselves. Having investigated this question using NIRS, we look forward to separate publications of these studies in the future.

\section{References}

1. H. Siesler, Y. Ozaki, S. Kawata, and H. M. Heise (ed.), "Near-Infrared Spectroscopy", 2002, Wiley-VCH Verlag, Weinheim, 1 - 3.
2. D. A. Burns and E. W. Ciurczak, "Handbook of NearInfrared Analysis", 2001, Marcel Dekker, New York, 1 - 9.

3. A. W. Brant, A. W. Otte, and K. H. Norris, Food Tech., 1951, $5,356$.

4. G. S. Birth and K. H. Norris, Food Tech., 1958, 12, 592.

5. M. Kumagai, H. Suyama, T. Sato, T. Amano, and N. Ogawa, J. Near Infrared Spectrosc., in press.

6. L. D. Hutcheson and R. S. Hughes, Appl. Opt., 1974, 13, 1395.

7. P. Cunniff (ed.), "Official Methods of Analysis of AOAC International", 16th ed., 5th Revision, 1999, AOAC International, USA.

8. P. Kubelka and F. Munk, Z. Tech. Phys., 1931, 12, 593.

9. J. N. Miller and J. C. Miller, "Statistics and Chemometrics for Analytical Chemistry", 4th ed., 2000, Pearson Education Ltd., England, 25.

10. M. Iwamoto and J. Uozumi, Nippon Shokuhin Kogyo Gakkaishi, 1985, 32, 685.

11. D. Bertrand, P. Robert, and W. Loisel, J. Sci. Food. Agric., 1985, 36, 1120. 